\title{
Aerial mapping of rice crops using mosaicing techniques for vegetative index monitoring
}

\author{
Juan P. Rojas B. *, Carlos A. Devia P.*, E. Petro ${ }^{\dagger}$, Carol Martinez *, Ivan F. Mondragon B*, D. Patino* MC. \\ Rebolledo ${ }^{\dagger \ddagger}$ and J. Colorado * \\ ${ }^{*}$ School of Engineering, Pontificia Universidad Javeriana, Bogota, Colombia \\ ${ }^{\dagger}$ The International Center for Tropical Agriculture -CIAT, Agrodiversity, Palmira, Colombia \\ ${ }^{\ddagger}$ CIRAD, AGAP-Pam, Montpellier, France
}

\begin{abstract}
In Colombia up to $40 \%$ of yield variability is due to the effects of climate variations. Rapid phenotyping methods are needed to properly assess the crop and improve production rates. In this paper, we propose to focus on developing a noninvasive system for speeding up monitoring tasks in rice crops. Unmanned Aerial Vehicles are used to gather multispectral visual information for high-throughput crop monitoring. Georeferenced digital surface models of the crop are generated based on image mosaicing techniques to allow for the autonomous computation of several vegetative indices. This paper presents the implemented system (hardware and software) and a field report of experiments carried out at different crop growth stages.
\end{abstract}

\section{INTRODUCTION}

Unmanned Aerial Vehicles (UAVs) are the new technological step for crop monitoring. They enable the acquisition of crop images in high resolution compared to the ones traditionally captured by satellites. Additionally, UAVs are able to obtain several information of the state of the crop autonomously by using a wide range of sensors onboard, such as, RGB, multispectral or thermal cameras, Lidars, DGPS, among others.

UAVs have helped to cover different problems in agriculture, e.g., crop parcels detection [1], fruit detection [2], crop variable measurement [3], [4], crop yield improvement [5], and crop mapping [6], [7]. In Colombia, most farmers are currently aware about the potential of using UAVs for improving crop yield performance [8], [9], however, most of the UAV-based solutions are developed overseas, constraining the rapid adoption of these technologies mostly for small farmers [10], [11].

In this paper, we propose the integration of a low-cost system using UAVs equipped with RGB-NIR cameras with computing methods for acquiring high-resolution and georeferenced images of the crop. Our goal is twofold: (i) creating a digital surface model of the crop by means of image mosaicing methods, and (ii) computing vegetative indices from the assembled crop map to estimate several crop variables, such as: biomass, nitrogen content or water stress. This paper primarily focuses on the former: methods for mapping rice crops to enable farmers to carry out comprehensive analysis of the crop biomass.

Regarding the aerial mapping of crops, in [12], a Phantom 2 UAV with a high-resolution RGB multispectral camera
(Micasense) was used to detect sheath blight fungus disease of rice. The Pix4D software was used to create geo-referenced 2D map and 3D models of the terrain and to calculate different vegetation index, such as NDVI. Others [13], [14], have explored the use of UAVs for rice nitrogen status estimation or chlorophyll quantification [15] using hyperspectral sensors.

A common point of the above mentioned approaches is that all agree on the potential benefits of using UAVs to improve the agricultural sector. However, there is no evidence that those approaches can be generalized to different types of farming methods and scaled to all stages of the crop. Attempting to develop an unique method for crop analysis and phenotyping that can be applied to all crop growing stages is a major challenge, since most of the physical and morphological characteristics of the plants can dramatically change along time. Additionally, most solutions rely on expensive commercial software to estimate the state of the crop, difficulting smallfarmers of integrating rapid phenotyping technology.

Moreover, a proposal of a complete system (hardware and software) for crop monitoring is also missing in the state of the art. Whether the crop analysis is conducted off-line, the results are highly-dependent on the type of hardware used in the tests. In this sense, our proposed system shows hardware and software that can deal with all the stages required for monitoring rice crops and without relying on commercial software. The paper is organized as follows: Section III describes the challenges of monitoring rice crops using UAVs and imagebased data. Section III presents the proposed hardware and software system. Section IV presents a field report of the flights that were conducted, whereas conclusions and direction of future work are presented in Section V.

\section{Problem Statement}

In Colombia, $51 \%$ of the total area is planted in upland than lowland production system. Preliminary data for 2017 indicates that this trend increased to $53 \%$ However, in terms of the number of cropping seasons per year, there is much more in lowland than upland conditions. The difference between both crop system methods is the source and the availability of water. In lowland farming, a permanent lamina of water stays during all the cropping season, whereas in upland farming the rice is irrigated with rainwater. In this paper, we have applied 


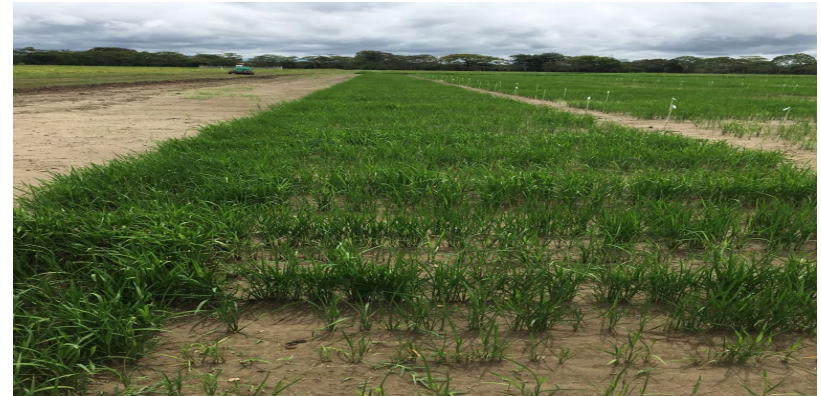

(a) Upland rice crop system

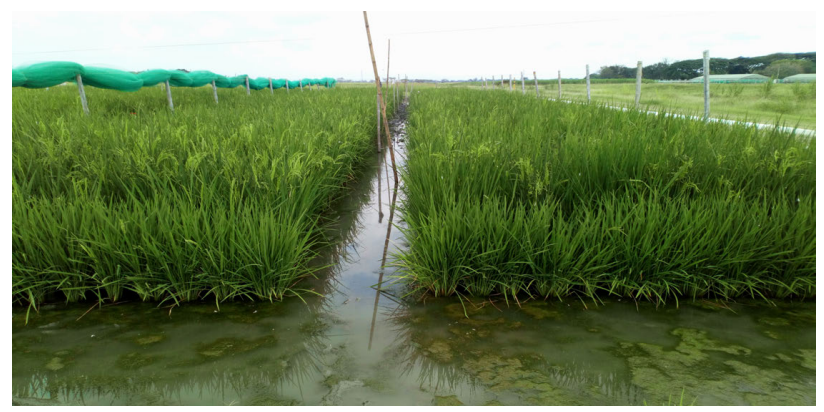

(b) Lowland rice crop system

Fig. 1. Production systems: lowland (transplanting) and ipland (direct seeding).

and tested our proposed mapping tool for both type of crop systems. Figure 1 depicts the crop scenarios.

Typical methods to monitor rice crops are based on destructive sampling (samples of the plant are manually harvested to the ground level and taken to the laboratory to analyze its state). To avoid this, non-invasive image-based methods have recently emerged in the past years. In this sense, UAV platforms with on-board visual sensing capabilities can play an important role for facilitating a high-throughput monitoring of the entire crop.

Developing a UAV-based system for measuring the status of these two types of crop systems presents multiple challenges. The UAV platform requires enough autonomy to cover the crop area, sufficient storage capacity to capture high-resolution data, and low-weight sensors onboard. Additionally, the integration of the aforementioned features requires an interplay between hardware and software to ensure the reliability of the data and a seamless experience for the end-user. In addition, the challenge of measuring and analyzing both crop systems at the same time aimed at determine phenotyping algorithms that can be applied no matter the conditions.

In terms of image analysis, as was shown in Section[ computer vision techniques have been widely applied for crop analysis. Nevertheless, the analysis of the image data of rice crops presents important challenges. The data is captured outdoors from a flying system. Therefore, motion can create blurred images; and weather conditions can affect the quality of the data (sunny, cloudy), especially in lowland crops, where water reflectance can change the appearance of the image.

Another important factor is the appearance of the plant as it grows. Fig. 2 shows how the features change in three representative stages of the crop: vegetative, reproductive, and ripening stages. In the vegetative stage occurs the development of tillers, the number of leaves increase, as well as the height of the plant. In this stage, the green color is predominant (see Figs. 2(a) and 2(d)). In the reproductive stage, panicle formation starts and thus yellow features appear in the images (see Figs. 2(b) and 2(e)). Finally, in the ripening stage, the flowering, the grain filling, and the maturation of the plant occur. In this stage, the yellow color is predominant and the parcel can barely be distinguished from panicles, grains and senescent leaves predominates (see Figs. 2(a) and 2(d)).

In Fig. 2 is possible to observe that as long as the plant grows, it turns more difficult using RGB images to separate the parcels for distinguishing between plants and background. Therefore, general assumptions about the color, size of the plant and the color of the soil will not always work. Considering all mentioned restrictions, the system presented in this paper is conceived as an effort to automate the monitoring of rice crops using a non-destructive method.

\section{PROPOSED SySTEM}

Figure 3 shows the architecture of the developed system. It is divided into four modules:

- Module 1: Hardware for multispectral sensing

- Module 2: Flight planning

- Module 3: Multispectral image processing for georeference map construction

- Module 4: Multispectral image processing for crop state estimation

\section{A. Hardware for multispectral sensing}

Two different UAVs have been integrated and tested in the proposed system: The ASCTEC Pelican and the ASCTEC Hummingbird (see Fig. 3). The ASCTEC Pelican is equipped with a Mastermind board (for image processing and data acquisition); the ASCTEC autopilot; and the Tetracam ADCLite multispectral camera. This camera captures visible light wavelengths longer than $520 \mathrm{~nm}$ and near-infrared (NIR) wavelengths up to $920 \mathrm{~nm}$. The multispectral camera is located in the bottom of the drone and aligned with the center of mass.

On the other hand, the ASCTEC Hummingbird was equipped with the the ASCTEC autopilot and the Parrot Sequoia multispectral camera. This camera comes with 4 different sensor to capture image in different wavelengths: red, green, NIR and Red-Edge.

\section{B. Flight Planning}

The ASCTEC UAVs come with GPS way point navigation capacity. A standard computer is used as ground station to run the Graphical User Interface that comes with the ASCTEC autopilots. This interface allows to control the UAV (autonomous take-off and landing; and waypoint navigation) and provides information about the state of the UAV.

An additional module was created for planning the image capture process [16]. The developed software creates a GPS grid with the coordinates of the places where the images 


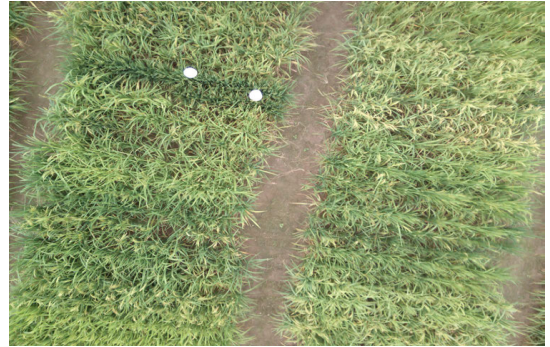

(a) Upland vegetative stage - no panicles, only leaves are observed.

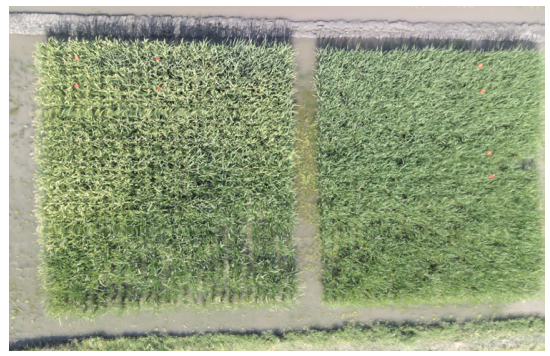

(d) Lowland vegetative stage

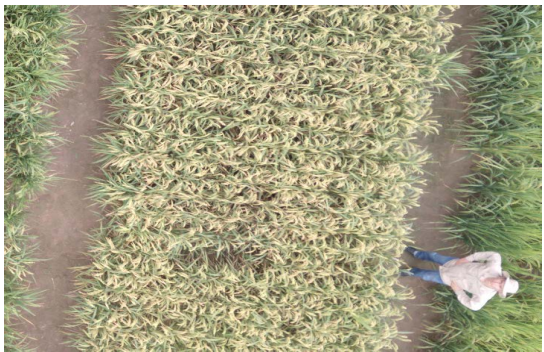

(b) Upland reproductive stage - the reproductive stage ends with the flowering. A few panicles should be observed.

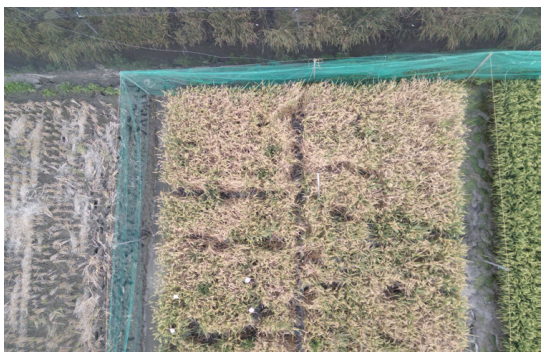

(e) Lowland reproductive stage

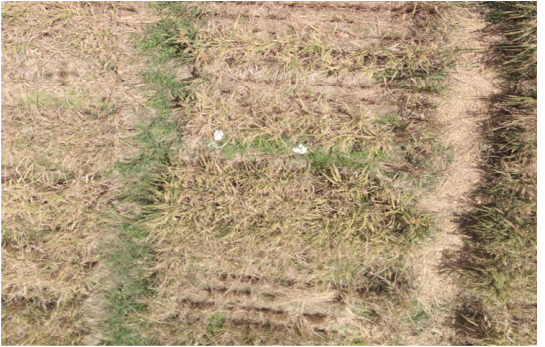

(c) Upland ripening stage

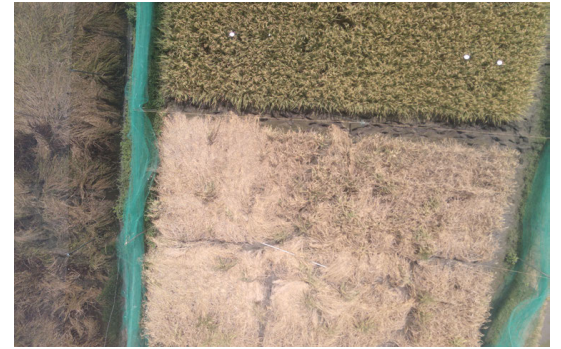

(f) Lowland ripening stage

Fig. 2. Changes in appearance as the plant grows. In the upland and lowland methods to grow rice, the appearance of the plant changes between stages. In the vegetative stage, Figs. 2(a) and 2(d) the green color is predominant. In reproductive stage, Figs. 2(b) and 2(e), panicle formation starts and yellow features appear, but parcels can still be differentiated. In ripening stage, the yellow color is predominant, and parcels can not be distinguished.

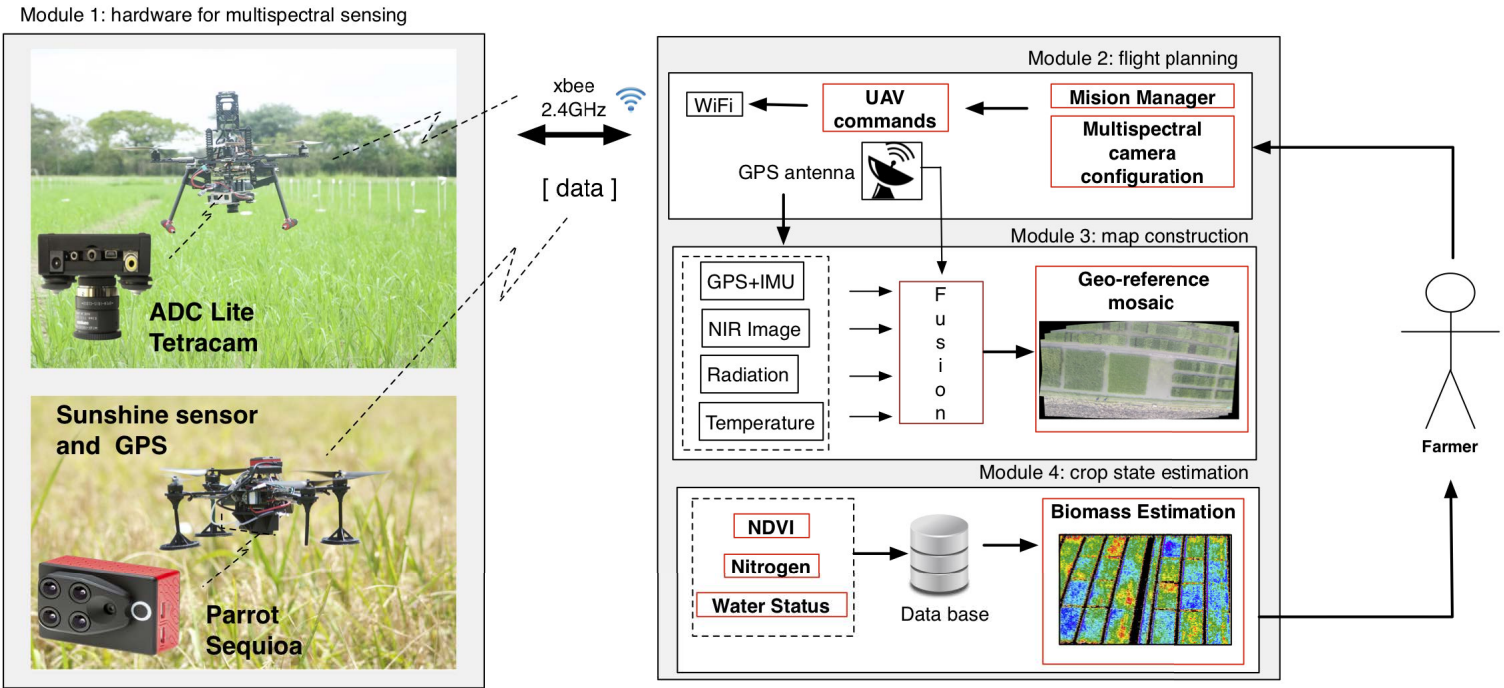

Fig. 3. System architecture

must be captured to ensure: the coverage of the terrain, the appropriate image resolution, and enough overlapped between images for generating a mosaic.

The photo planner algorithm was presented in [16]. It is based on geometric parameters, such as the camera intrinsic parameters, the work area, and the height of the drone. This information is used to generate the cartesian points. In this model it is assumed that the grid evolves from the lower right corner and moves to the left corner, and from the bottom of the area to the top. Therefore, the estimation of the cartesian grid points are based on Eq. 1. In this equation, $p_{x}$ and $p_{y}$ correspond to the overlap in the $\mathrm{x}$ and $\mathrm{y}$ axes of the work area, $u$ and $v$ are the width and height seen by the camera in meters, $y_{i-1}$ and $x_{i-1}$ are the previous calculated positions, and $y_{i}$ and $x_{i}$ the new estimated positions on the plane. This model is iterative, that means that in every iteration create a new position based on the calculated before. Also is important to note that in the code every odd column must be flipped in order to get a continuous path.

$$
\left[\begin{array}{l}
x_{i} \\
y_{i}
\end{array}\right]=\left[\begin{array}{l}
x_{i-1}+u\left(-1+p_{x}\right) \\
y_{i-1}+v\left(-1+p_{y}\right)
\end{array}\right]
$$

The photo planning algorithm includes a feature that allows to estimate the altitude to fly $h_{U A V}$, based on the camera's 


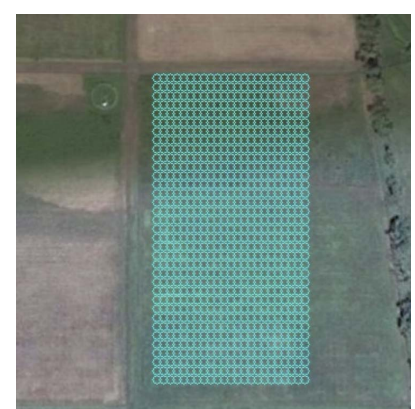

(a) Overlap of $60 \%$

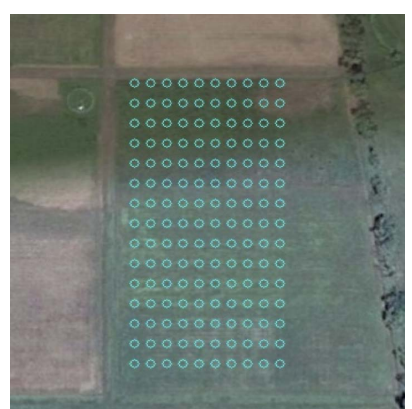

(b) Overlap $10 \%$
Fig. 4. Grids generated by the photo planning algorithms with different percentages of overlap. The images correspond to the flights conducted in Villavicencio zone (upland rice crop). The flight area was $100 \mathrm{~m} \times 200 \mathrm{~m}$, the UAV's height was $12 \mathrm{~m}$, and the camera FOV was $48.50 \times 61.90$. Fig. 4(a) shows the grid generated when the percentage of overlap was $60 \%$ in both directions. Fig. 4(b) the percentage of overlap was the $10 \%$.

field of view in each axis $\left(\boldsymbol{\theta}=\left\{\theta_{x}, \theta_{y}\right\}\right)$ and the desired image resolution $\mathbf{d}_{\text {cam }}$, as shown in Eq. 2 .

$$
h_{U A V}=\frac{\boldsymbol{\theta}^{\boldsymbol{T}} \cdot \mathbf{d}_{\mathbf{c a m}}}{2}
$$

where $\mathbf{d}_{\text {cam }}$, corresponds to the image size in pixels, multiplied by the desired resolution in meters per pixel.

Figure 4 shows some of the grids for the flight trials in Villavicencio. The image on the left corresponds to the grid created when an overlapped of $60 \%$ is used. The one on the right corresponds to an overlap of $10 \%$.

When the cartesian grid is calculated, then the global coordinates of those points must be estimated. Equations 3 and 4 were used to make that conversion.

$$
\begin{gathered}
\phi_{f}=\arcsin \left(\left(\sin \left(\phi_{0}\right) \cos (\delta)\right)+\right. \\
\left(\cos \left(\phi_{0}\right) \sin (\delta) \cos (\theta)\right) \\
\theta_{f}=\theta_{0}+\operatorname{atan} 2\left(\sin (b) \sin (\delta) \cos \left(\phi_{0}\right),\right. \\
\left.\cos (\delta)-\left(\sin \left(\phi_{0}\right) \sin \left(\phi_{f}\right)\right)\right)
\end{gathered}
$$

Where $\phi_{0}$ and $\theta_{0}$ are the latitude and longitude of the first point (known global coordinates); $\phi_{f}$ and $\theta_{f}$ are the estimated latitude and longitude of the final point, given the bearing $(b)$ and the angular distance $\delta$. Knowing that $\delta=\frac{\text { Traveled_distance }}{\text { Earth_radius }}$. This process is iterative, because each new global point is calculated based on the distance between the grid points and the initial value of bearing.

The defined bearing is very important because it gives the global direction of the grid. To be more punctual, when the bearing is 0 , the calculated global coordinate is going to point to the north pole in a perfect line; and when the bearing is 90 , the estimated points are going to be parallel to the equator line. However, depending on the part of the earth where you are the direction could change. The bearing of a zone is calculated using the Eq. 5, for this estimation two more coordinates are required. This two new coordinates could be related to the top and bottom of the crop area.

$$
b=\operatorname{atan} 2\left(\sin (\Delta \theta) \cos \left(\phi_{2}\right), \cos \left(\phi_{1}\right) \cos \left(\phi_{2}\right) \cos (\delta \phi)\right)
$$

\section{Multispectral image processing for geo-reference map con- struction}

For creating the image mosaic and geo-referencing the images, the metadata embedded in the images must be extracted. When the images are capture with the ADC-lite camera (shown in Fig. 3), the metadata is extracted from the log file generated by the drone. However, with the Parrot Sequoia camera, the images contain two different elements that have to be handled. The first is the exiv tag that contains: the original raw name of the image and the GPS coordinates where the image was taken. The second element corresponds to the $x m p$ tag that contains: the irradiance sensor measurements; the orientation of the sunshine sensor, at the moment of the shot; and the extrinsic and intrinsic parameters (orientation of the camera when the photo was taken, and $K$ matrix), and the focal length in $\mathrm{mm}$ of the four cameras (red, green, near infrared and red edge).

The library used to extract the information from the Sequoia was the exiv2 [17]. When all the metadata is extracted from the images, a *.csv file is generated with all the information.

1) Image mosaic: After the images are ordered, the process of constructing the image mosaic begins. Figure 5 describes the different stages of the algorithm required for registering two images.

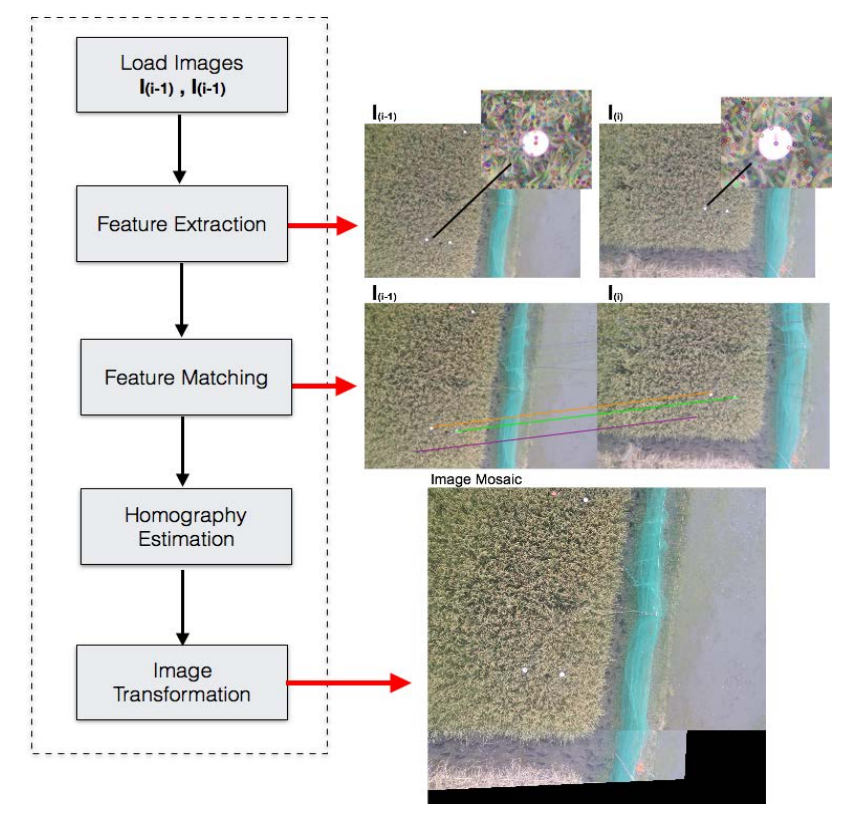

Fig. 5. Flow diagram of the algorithm used to create the mosaics of rice crops.

The first stage is the feature extractor. Features are extracted in both images (the reference image $\mathbf{I}_{(i-1)}$, and the current image $\mathbf{I}_{(i)}$ ). For this application two different algorithms were implemented (SURF [18] and the ORB [19] ), in order to test which algorithm provides better results for the application, in terms of execution time and quality of the generated mosaic (which is the best descriptor of rice crops). 
The second stage is the feature matching. The FLANN (Fast Library for Approximate Nearest Neighbors) algorithm [20] was implemented and used to match features between $\mathbf{I}_{(i-1)}$ and $\mathbf{I}_{(i)}$. The algorithm returns possible feature matches which are filtered by the euclidean distance, only matches with a distance $<1.5$, are considered.

Once, there are more than four well-matched points. The RANSAC (Random Sample Consensus) algorithm [21], [22], is used for estimating the homography $\mathbf{H}$, which transforms points in image $\mathbf{I}_{(i)}$ to the coordinate frame of image $\mathbf{I}_{(i-1)}$. Once another image arrives, e.g. $\mathbf{I}_{(i+1)}$, the previously mentioned process is repeated, but this time between images $\mathbf{I}_{(i)}$ and $\mathbf{I}_{(i+1)}$.

Therefore, for every pair of images, a homography $\mathbf{H}$ is estimated. Thus, consecutive homographies are concatenated to allow the transformation of the current image into the coordinate frame of the first image.

2) Geo-referencing: The process of geo-referencing refers to relate a global coordinate system to each pixel of an image. This will allow to conduct distance measurements on the image and navigation at global scale. To generate a geo-reference image it is important to define the type of transformation to use, and to properly select the control points. They should not be aligned and must be scattered along the work area, to get a better relation between the real world coordinates and the pixels that represent them.

To select the transformation, there are five widely used transformations. The affine transformation, related to a polynomial of $1^{\text {st }}$ order, requires the used of minimum 3 known global coordinates in the image to solve the system. This homogeneous transformation allows to preserve straight lines. In general affine transformation is used for general needs (translations, scale, and rotations). However, if the plane of the image must be adjusted to a more complicated plane that contain curves, polynomials of $2^{\text {nd }}$ or bigger orders, must be used [23].

In this paper, an affine transformation is used. We are working on flat terrains, and because the distance between crops is less than a kilometer, it is possible to assume that the coordinates change linearly and there is not need to reexpress the coordinates in a more complex plane.

The process for geo-referencing the image consists in measuring 3 or more global coordinates and apply two linear equations shown in Eq. 6. These equations have as unknown elements the variables $A, B, C, D, E$, and $F$; which correspond to the nine elements of the affine transformation [23]. $A$ and $E$ are the width and height of each pixel in map units, $B$ and $D$ are the rotation components, and $C$ and $F$ represent the latitude and longitude of upper left pixel of the image [23]. $x$ and $y$ represent the pixel coordinates of a control point. To get this unknown variables, three or more ground control points (GCPs) are needed. This GPCs are used to complete the system of equations shown in Eq. 7 Where $\mathbf{v}$ is a column vector of $6 \times 1$ that represents the latitudes and longitudes of the real world, $\mathbf{M}$ is a matrix of $6 \times 6$ that contains the pixel coordinates of the map, and $\mathbf{u}$ is a vector of $6 \times 1$ that contains the nine elements of the affine transformation. Therefore, $\mathbf{u}=\mathbf{M}^{-\mathbf{1}} \mathbf{v}$.

$$
\begin{gathered}
{\left[\begin{array}{l}
\phi \\
\lambda
\end{array}\right]=\left[\begin{array}{l}
A x+B y+C \\
D x+E y+F
\end{array}\right]} \\
\mathbf{v}=\mathbf{M u}
\end{gathered}
$$

Once the affine transformation is known, Eq. 6 can be used to estimate the latitude $\pi$ and longitude $\lambda$ related to a pixel coordinate $(x, y)$.

\section{Multispectral image processing for crop state estimation}

The crop state estimation is related to the measurement of the stress in the plant caused by low water levels, high levels of $p H$ in the soil, saline soils, plague, little sun radiation, among others. In this project, we propose the measurement of biomass, nitrogen levels, and water stress, as variables that allows to know the crop state, and let us know how it evolves over time.

The approach used to measure the biomass is to calculate from the multi-spectral images, different vegetation indices: SR (Simple Ratio), NDVI (Normalized difference vegetation), GNDVI (Green Normalized Difference Vegetation Index), CTVI, SAVI (Soil Adjusted Vegetation Index), DVI ( Difference Vegetation Index), and MSAVI (Modified Soil-adjusted Vegetation Index). Then, with the reflectance values of the indices; and with the Ground Truth data, obtained from the harvested areas, a multi-variable regression is conducted to calculate the constants. With them, a model that let us estimate the biomass from the image data, is created.

\section{RESUlTS}

\section{A. Experimental Setup}

In the course of 2017, 24 flights were conducted. The flights were carried out in two rice farms of the Center of International Agriculture -CIAT that have two different methods of growing rice: lowland (flooded rice paddies), and upland (dry soils). Measurements from the crop were obtained, in both farms, during three stages of rice growth: vegetative, reproductive, and ripening.

Figure 6 shows the trajectory followed by the UAV in one of the flights conducted in Villavicencio.

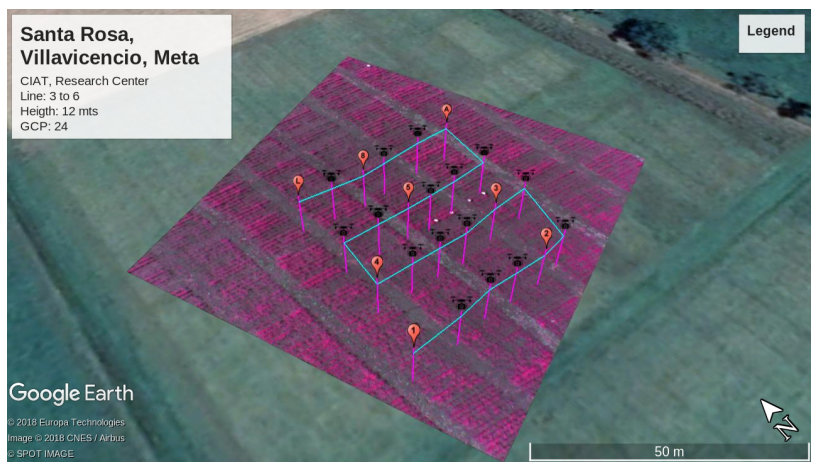

Fig. 6. UAV path followed during Villavicencio's tests. The multispectral view of one segment of the crop is shown.

After the flights, the metadata of each picture was extracted, and vegetative indices of each image and the RGB mosaic 


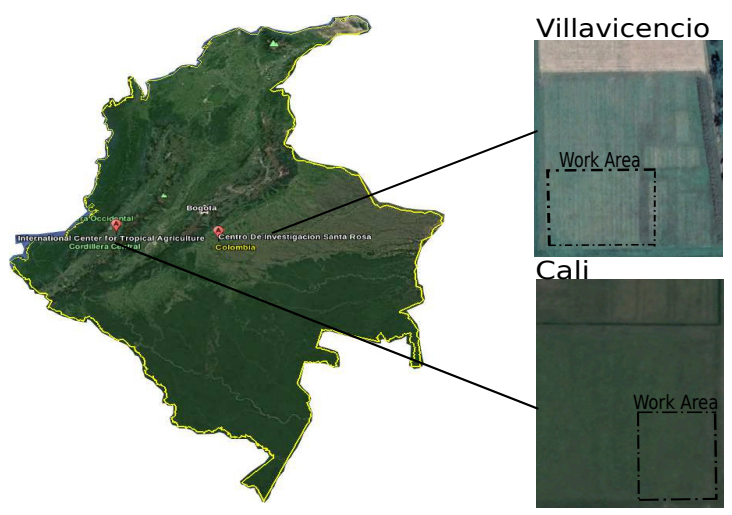

Fig. 7. Place of the Experiments. The flights were carried out in two rice farms of the Center of International Agriculture -CIAT. Villavicencio, Meta, at the research center of CIAT in Santa Rosa (upland, dry soils wroth method) and Palmira, Cali in the principal farm of CIAT (lowland, flooded rice paddies).

were generated. The different components of the algorithm were tested using a laptop with an $I 7$ intel processor, $32 G B$ of RAM, GPU, and NVIDIA980GTX. The algorithms are based on ROS and OpenCV libraries.

1) Villavicencio, Meta (upland): The first experiments of the year were carried out in Villavicencio, Meta, at the research center of CIAT in Santa Rosa. Images of two types of rice: IR64 and LINE23, in vegetative, reproductive, and ripening stages, were taken. Examples of the images can be seen in Fig. 2, first row. Per stage, two flights were conducted in the morning, and two in the afternoon.

The covered area was $\approx 100 \mathrm{~m}^{2}$. The first flight of every pair was conducted at $12 \mathrm{~m}$ height, and the second at $15 \mathrm{~m}$. The days of the tests were sunny days, with few clouds, and soft winds. Figure 7 shows the work area, and Fig. 6 shows the trajectory followed by the UAV in one of the flights.

After capturing images of the crop, measurements of the crop were manually obtained. A lineal meter of the crop, previously marked by their GPS coordinates, is harvested to the ground level and taken to the laboratory to extract the rice matter to obtain the water content, the chlorophyll level, and the nitrogen level of the plant. This data is used as Ground Truth for testing the crop state estimation algorithms developed in the project (which are out of the scope of this paper).

2) Palmira, Cali (lowland): The final flights of the year were carried out in Palmira, Cali in the principal farm of CIAT. The flights were were conducted over a plot with water bed growth method (lowland). Images of two types of rice production system: IR64 and LINE23, in vegetative, reproductive, and ripening stages, were taken. Examples of the images captured in these tests can be seen in Fig. 2, second row.

Per stage, three flights were conducted in the morning, and three in the afternoon. The covered area was $\approx 80 \mathrm{~m}^{2}$. The first flight in every stage, was at $12 \mathrm{~m}$ height, and the other five flights were at $15 \mathrm{~m}$. The days of the tests were sunny days, with few clouds, and strong winds. The map of the work area can be see it in figure 7

As with the flights conducted in Villavicencio, measure-

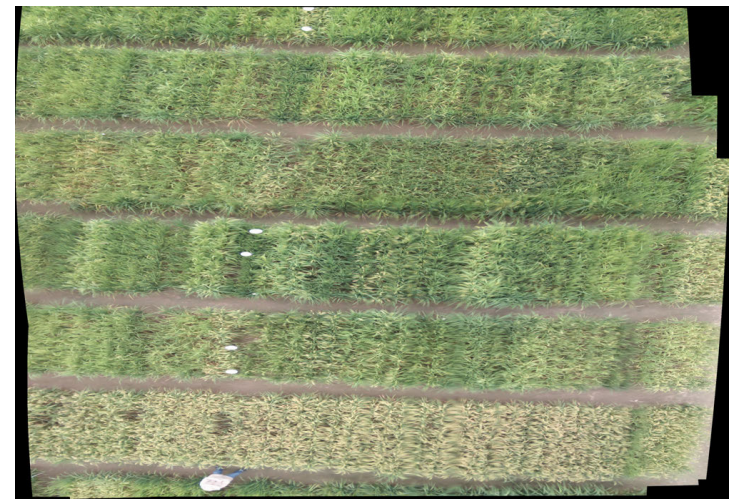

(a) Vegetative stage, Villavicencio

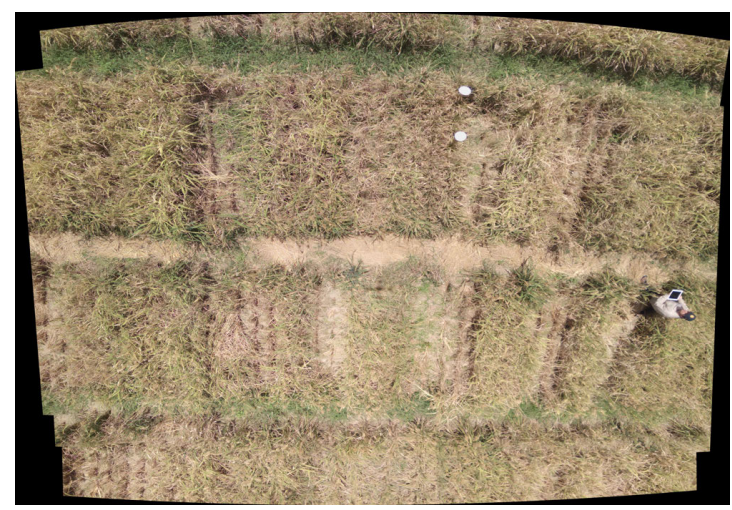

(b) Ripening stage, Villavicencio

Fig. 8. Image Mosaics from Upland Crop, Villavicencio. The mosaics correspond to images captured in flights conducted at different stages of the crop (vegetative and ripening). White circles in the image represent the areas of the crop were ground truth data was gathered (manual harvested samples sent to laboratory analysis).

TABLE I

DATA OF THE MOSAICS FOR UPLAND CROP, VILlaViCENCIO

\begin{tabular}{|c|c|c|c|}
\hline \multicolumn{5}{|c|}{ Vegetative stage } \\
\hline & Time $(\mathrm{seg})$ & \# of Images & $\%$ Overlap \\
\hline SURF & 8.6501 & 5 & 70 \\
\hline ORB & 7.1308 & 5 & 70 \\
\hline \multicolumn{5}{|c|}{ Ripening stage } \\
\hline & Time $(\mathrm{seg})$ & \# of Images & \% Overlap \\
\hline SURF & 9.3703 & 4 & 80 \\
\hline ORB & 9.1220 & 4 & 80 \\
\hline
\end{tabular}

ments of the crop were manually obtained and taken to the laboratory, to obtain ground truth data of the state of the plant.

\section{B. Image Mosaic}

The mosaic were generated for lowland and upland growing methods using the ORB and SURF feature extractors. Fig. 8 shows the mosaic of the flights conducted in Villavicencio zone. These mosaics correspond to two of the stages of the crop (vegetative and ripening). White circles in the image represent the areas of the crop were ground truth data was gathered (manual harvested samples were sent to a laboratory for analysis).

Table I compares the results of generating the mosaics using two different methods of feature extraction (SURF and ORB). 


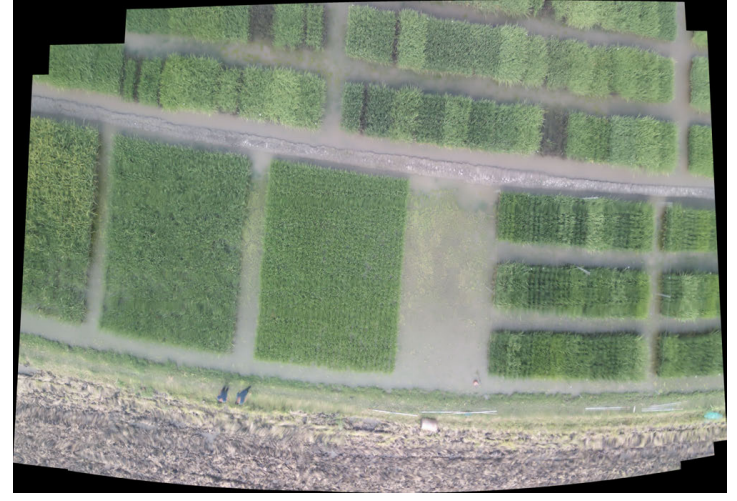

(a) Vegetative stage, Palmira

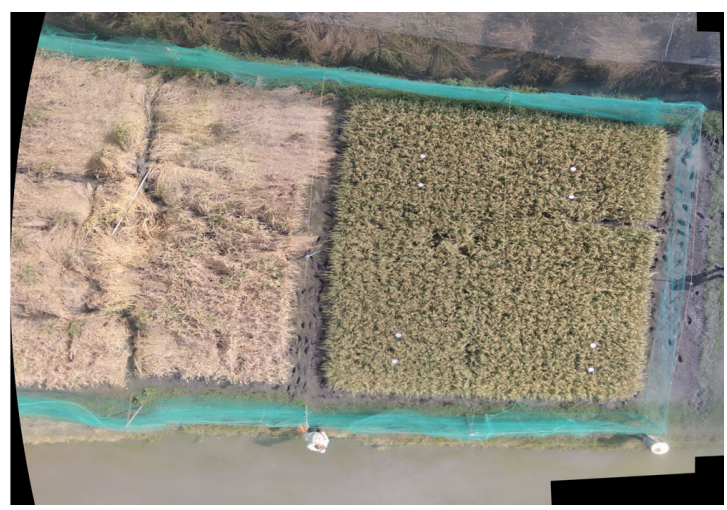

(b) Ripening stage, Palmira

Fig. 9. Image Mosaics from Lowland Crop, Palmira. The mosaics correspond to images captured in flights conducted at the vegetative and ripening stages of the crop.

TABLE II

Data of the Mosaics for Lowland Crop, Palmira

\begin{tabular}{|c|c|c|c|}
\hline \multicolumn{4}{|c|}{ Vegetative stage } \\
\hline & Time (seg) & \# of Images & $\%$ Overlap \\
\hline SURF & 9.2101 & 7 & 80 \\
\hline ORB & 8.9660 & 7 & 80 \\
\hline \multicolumn{4}{|c|}{ Ripening stage } \\
\hline & Time (seg) & \# of Images & $\%$ Overlap \\
\hline SURF & 13.9224 & 10 & 70 \\
\hline ORB & 13.0016 & 10 & 70 \\
\hline
\end{tabular}

On the other hand, Fig. 9 shows the mosaics of the flights conducted in Palmira, Cali. The mosaics correspond to the vegetative and ripening stages of the crop. Table II compares the results of generating those mosaics using the SURF and ORB feature extraction methods.

In general terms both algorithms for feature extraction (SURF and ORB) behaves similarly. There is not difference on the quality of the mosaics that were generated with them. In terms of execution time, from the tables, it is possible to see that ORB has a better performance than SURF. Nevertheless, the small difference in time is not relevant for the the application presented in this paper. Therefore, both algorithms could be use for generating mosaics of rice crops.

\section{Vegetation Indices}

Vegetation indices are extracted from the images captured in the flights. Figure IV-C shows different indices calculated from representatives images of the flights conducted in Villavicencio and Palmira that correspond to different stages of the crop (Villavicencio, ripening stage; and Palmira, vegetative stage).

The following vegetation indices were estimated NDVI, GNDVI, DVI, TVI, MSAVI. In Fig. 10(a) and Fig. 10(b), it can be seen that the appearance of the crop is black. Which means that the leaves had a low level of reflectance. This is related to the chlorophyll level that each plant has. In the ripening stage, this value is low. Conversely, in Figs. 10(f) and $10(\mathrm{~g})$, the appearance of the crop is brighter. This is due to the high level of chlorophyll in their leaves, typical in the vegetative stage.

Therefore, with the different vegetative indices, it is possible to obtain information about the changes of the crop over time.

On the other hand, Fig. 11 shows a multispectral mosaic where different vegetation indices were calculated. Table III shows their values.

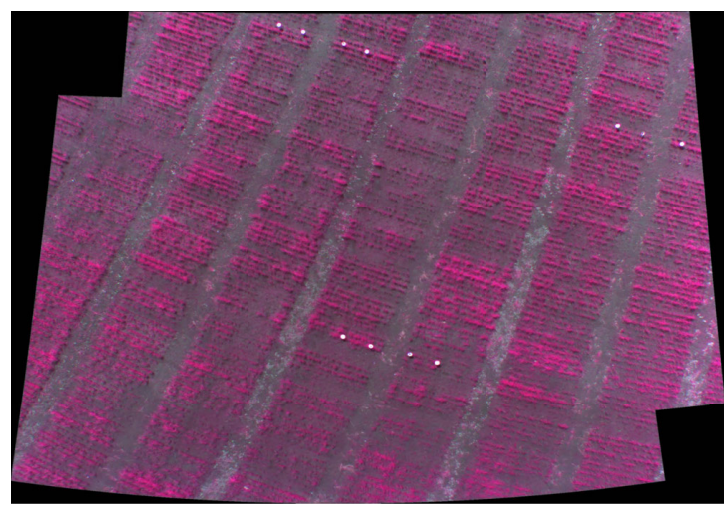

Fig. 11. Multi-spectral mosaic for crop analysis. The image correspond to one the flights conducted in Villavicencio, during the vegetative stage of the crop.

TABLE III

VEGETATION INDICES FROM THE MULTI-SPECTRAL MOSAIC OF FIG. 11

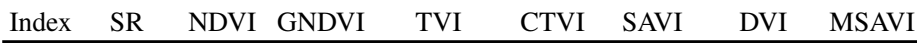

$\begin{array}{lllllllll}\text { Value } & 3.7972 & 0.1128 & 0.2873 & -13.6882 & 0.6222 & -0.995 & -0.0804 & 0.1832\end{array}$

\section{Geo-referencing}

Fig. 12 shows a geo-referenced mosaic of the crop in Palmira Cali. For generating and testing the geo-referencing algorithm, ten manually measured GPS points are used. These points are shown in the figure (red points). Points 2, 6 and 10 were used as ground control points (GCP) for estimating the affine model. With that model, the geo-referenced mosaic was created.

To test the result, points $1,3,4,5,7,8$, and 9 , corresponding to some of the vertices of each plot, were used as ground truth data (GT). The coordinates of these points was compared to the one provided by the geo-referencing algorithm. Table IV shows the comparison. 


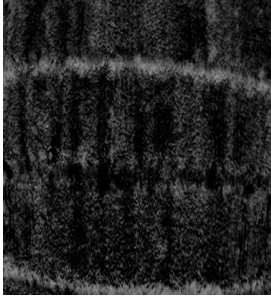

(a) NDVI, Villavicencio

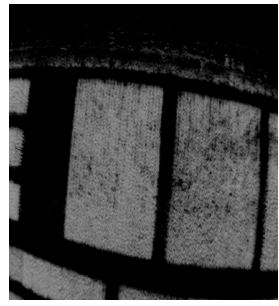

(f) NDVI, Palmira

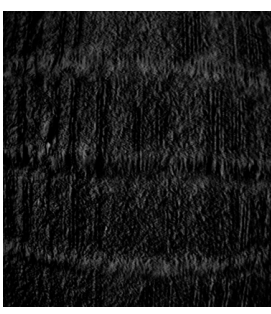

(b) GNDVI, Villavicencio

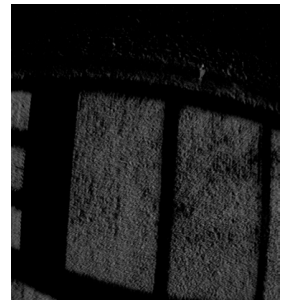

(g) GNDVI, Palmira

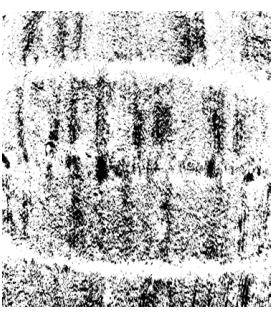

(c) DVI, Villavicencio

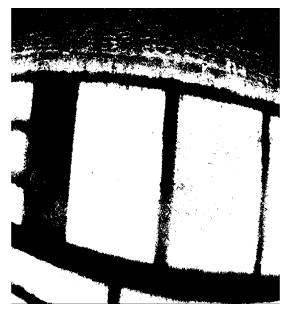

(h) DVI, Palmira

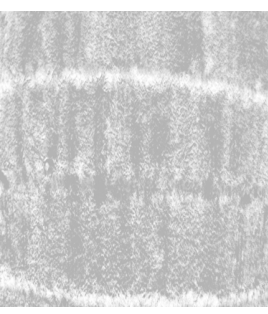

(d) TVI, Villavicencio

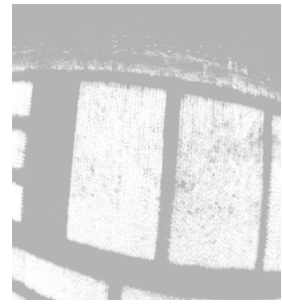

(i) TVI, Palmira

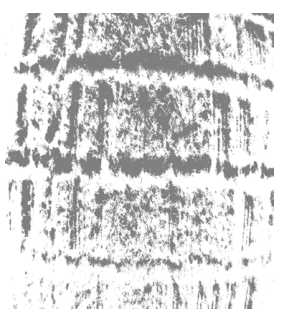

(e) MSAVI, Villavicencio

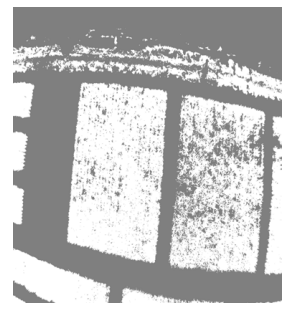

(j) MSAVI, Palmira

Fig. 10. Vegetation indices extracted from representatives images of the flights conducted in Villavicencio (ripening stage) and Palmira (vegetative stage). In Figs. 10(a) and 10(b), leaves had low level of reflectance. This is because in ripening stage, chlorophyll levels are low. Figs. 10(f) and 10(g) higher levels of reflectance. This is because in vegetative stage, chlorophyll levels are high.

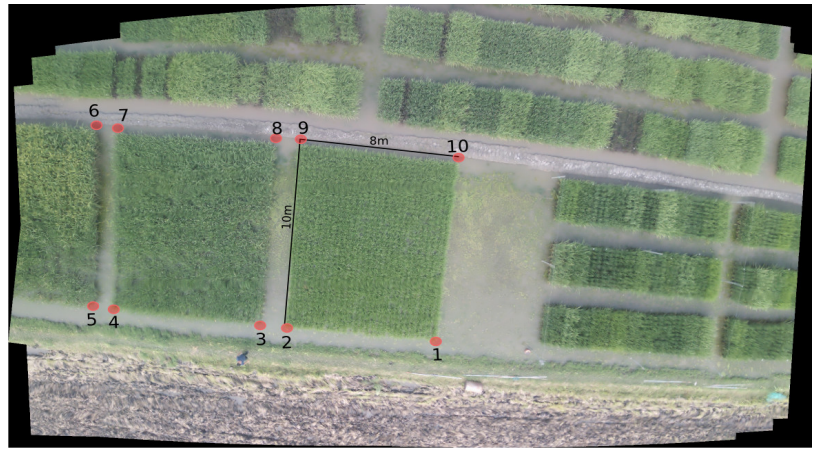

Fig. 12. Geo-referenced mosaic of the crop in Palmira Cali. Red points represent 10 manually measured GPS points. Points 2,6 and 10 are used as ground control points (GCP) for estimating the affine model. The other points are used as confirmation points.

Additionally, the distance between points was measured using the model of the haversine formula. Table $\mathrm{V}$ compares the distances obtained with the GT points with the ones estimated by the geo-referencing algorithm. It is important to mention that the measurements were done with a normal GPS.

TABLE IV

COMPARISON OF GPS COORDINATES

\begin{tabular}{|l|c|c|c|}
\hline & Measured $\left(^{\circ}\right)$ & Estimated $\left(^{\circ}\right)$ & Error $(\mathrm{m})$ \\
\hline PT1 & $3.501200 \mathrm{~N} 76.365972 \mathrm{~W}$ & $3.501209386 \mathrm{~N} 76.3659659 \mathrm{~W}$ & 1.136 \\
\hline PT3 & $3.501217 \mathrm{~N} 76.352006 \mathrm{~W}$ & $3.501216530 \mathrm{~N} 76.35202171 \mathrm{~W}$ & 1.744 \\
\hline PT4 & $3.501156 \mathrm{~N} 76.365864 \mathrm{~W}$ & $3.501150499 \mathrm{~N} 76.36587214 \mathrm{~W}$ & 1.091 \\
\hline PT5 & $3.501158 \mathrm{~N} 76.365858 \mathrm{~W}$ & $3.501147421 \mathrm{~N} 76.36586375 \mathrm{~W}$ & 1.338 \\
\hline PT7 & $3.501139 \mathrm{~N} 76.365931 \mathrm{~W}$ & $3.501138220 \mathrm{~N} 76.36593916 \mathrm{~W}$ & 0.9098 \\
\hline PT8 & $3.501197 \mathrm{~N} 76.365975 \mathrm{~W}$ & $3.501193306 \mathrm{~N} 76.36596545 \mathrm{~W}$ & 1.140 \\
\hline PT9 & $3.501230 \mathrm{~N} 76.365915 \mathrm{~W}$ & $3.501245341 \mathrm{~N} 76.36591338 \mathrm{~W}$ & 1.715 \\
\hline
\end{tabular}

TABLE V

COMPARISON OF DISTANCES BETWEEN COORDINATES

\begin{tabular}{|l|c|c|c|}
\hline & Measured $(\mathrm{m})$ & Estimated $(\mathrm{m})$ & Error $(\mathrm{m})$ \\
\hline PT01 to PT10 & 10.0 & 7.068 & 2.932 \\
\hline PT01 to PT02 & 8.0 & 5.474 & 2.526 \\
\hline
\end{tabular}

\section{CONCLUSION AND FUTURE WORK}

We have presented results from different flight trials conducted in Colombia with the objective of acquiring visual information of the state of rice crops with two different methods of growth (upland and lowland). Image were captured at different stages of the crop in order to gather enough information to analyze the viability of estimating the state of rice crop with those images. Additionally, ground truth data was obtained directly from the crop.

In this paper, we presented the system (software and hardware) created for obtaining the above mentioned information. Additionally, we have shown results of the different modules. Currently, the developed system allows to generate the trajectory of the UAV, to ensure the acquisition of the required image data during the flight (ensuring an overlap between images). With the acquired data, the system generates a geo-referenced mosaic of the crop; and calculates different vegetation indices which can provide information about the state of the crop.

Current work is focused on analyzing the acquired information for estimating nitrogen levels and water status of the crop.

\section{ACKNOWLEDGEMENTS}

This work has been funded and sponsored by the research project entitled Desarrollo de una herramienta para la agricultura de precision en los cultivos de arroz: sensado del estado de crecimiento y de nutricion de las plantas usando un drone autonomo (Aerial sensing and monitoring of rice crop fields applying precision agriculture techniques). Funded 
by: COLCIENCIAS. ID 120371551916 and developed by the Pontificia Universidad Javeriana, Bogota, Colombia, and The International Center for Tropical Agriculture -CIAT, Agrodiversity, Palmira, Colombia. Additionally, the authors thank to the Vice Chancellor Office for Research for supporting this project under the ID: PPTA 6895.

\section{REFERENCES}

[1] L. Hongli, Y. Zhoumiqi, Z. Jinshui, and G. Shuai, "Highly efficient paddy classification using uav-based orthorectified image," in 2017 IEEE International Geoscience and Remote Sensing Symposium (IGARSS), July 2017, pp. 3230-3233.

[2] G. L. A. Carrijo, D. E. Oliveira, G. A. de Assis, M. G. Carneiro, V. C. Guizilini, and J. R. Souza, "Automatic detection of fruits in coffee crops from aerial images," in 2017 Latin American Robotics Symposium (LARS) and 2017 Brazilian Symposium on Robotics (SBR), Nov 2017, pp. 1-6.

[3] C. M. Gevaert, J. Suomalainen, J. Tang, and L. Kooistra, "Generation of spectral 2013;temporal response surfaces by combining multispectral satellite and hyperspectral uav imagery for precision agriculture applications," IEEE Journal of Selected Topics in Applied Earth Observations and Remote Sensing, vol. 8, no. 6, pp. 3140-3146, June 2015.

[4] J. Gago, C. Douthe, R. Coopman, P. Gallego, M. Ribas-Carbo, J. Flexas, J. Escalona, and $\mathrm{H}$. Medrano, "Uavs challenge to assess water stress for sustainable agriculture," Agricultural Water Management, vol. 153, pp. 9 - 19, 2015. [Online]. Available: http://www.sciencedirect.com/science/article/pii/S0378377415000293

[5] J. A. Arroyo, C. Gomez-Castaneda, E. Ruiz, E. M. de Cote, F. Gavi, and L. E. Sucar, "Uav technology and machine learning techniques applied to the yield improvement in precision agriculture," in 2017 IEEE Mexican Humanitarian Technology Conference (MHTC), March 2017, pp. 137143.

[6] T. Guo, T. Kujirai, and T. Watanabe, "Mapping Crop Status From an Unmanned Aerial Vehicle for Precision Agriculture Applications," ISPRS - International Archives of the Photogrammetry, Remote Sensing and Spatial Information Sciences, vol. XXXIXB1, no. September, pp. 485-490, 2012. [Online]. Available: http://www.int-arch-photogramm-remote-sens-spatial-inf-sci.net/ XXXIX-B1/485/2012/

[7] R. Khanna, M. Martin, J. Pfeifer, F. Liebisch, A. Walter, and R. Siegwart, "Beyond Point Clouds - 3D Mapping and Field Parameter Measurements using UAVs," IEEE 20th Conference on Emerging Technologies \& Factory Automation, pp. 5-8, 2015.

[8] Fedesarrollo, "Política Comercial Para El Arroz - Reporte Final," p. 58, 2013.

[9] J. Chica, Y. Tirado, and J. M. Barreto, "Indicadores de competitividad del cultivo del arroz en Colombia y Estados Unidos," Ciencias Agrícolas, vol. 33, no. 2, pp. 16-31, 2016.

[10] I. D. E. Gesti, F. Nacional, D. E. L. Arroz, F. Nacional, F. Nacional, F. Arrocero, F. Nacional, L. G. General, F. Nacional, F.-f. Nacional, and F. Tecnol, "Informe de gestión vigencia fondo nacional del arroz 2016," vol. 2016, pp. 1-52, 2016.

[11] J. Borrero, S. R. Mccouch, and W. Roca, "Estado actual del mejoramiento del arroz mediante la utilización de especies silvestres de arroz en ciat 1," Current, vol. 9, no. 1, pp. 10-17, 1998.

[12] J. Dongyan Zhang, Xingen Zhou, Jian Zhang, Linsheng Huang and Zhao, "Developing a small uav platform to detect sheath blight of rice," IGRASS, pp. 7-10, 2017.

[13] L. Yong, C. Tao, Z. Yan, T. Yongchao, C. Weixing, Y. Xia, W. Ni, H. Zheng, X. Zhou, T. Cheng, X. Yao, Y. Tian, W. Cao, and Y. Zhu, "Comparative analysis of vegetation indices, non-parametric and physical retrieval methods for monitoring nitrogen in wheat using UAV-based multispectral imagery Yong Liu, Tao Cheng, Yan Zhu, Yongchao Tian , Weixing Cao , Xia Yao *, Ni Wang National En," Ieee, pp. 7350-7353, 2016.

[14] J. Lu, Y. Miao, Y. Huang, W. Shi, X. Hu, X. Wang, and J. Wan, "Evaluating an unmanned aerial vehicle-based remote sensing system for estimation of rice nitrogen status," 2015 4th International Conference on Agro-Geoinformatics, Agro-Geoinformatics 2015, pp. 198-203, 2015.

[15] K. Uto, H. Seki, G. Saito, and Y. Kosugi, "Characterization of rice paddies by a UAV-mounted miniature hyperspectral sensor system," IEEE Journal of Selected Topics in Applied Earth Observations and Remote Sensing, vol. 6, no. 2, pp. 851-860, 2013.
[16] J. Rojas, C. Martinez, I. Mondragon, and J. Colorado, "Towards image mosaicking with aerial images for monitoring rice crops," in Advances in Automation and Robotics Research in Latin America. Springer, 2017, pp. $279-296$.

[17] E. ORG. (2017) C++ metadata library and tools exif, iptc and xmp metadata and icc profile. [Online]. Available: http://www.exiv2.org/ getting-started.html

[18] H. Bay, T. Tuytelaars, and L. Van Gool, "Surf: Speeded up robust features," in European conference on computer vision. Springer, 2006, pp. 404-417.

[19] E. Rublee, V. Rabaud, K. Konolige, and G. Bradski, "Orb: An efficient alternative to sift or surf," in Computer Vision (ICCV), 2011 IEEE international conference on. IEEE, 2011, pp. 2564-2571.

[20] M. Muja and D. G. Lowe, "Scalable nearest neighbor algorithms for high dimensional data," IEEE transactions on pattern analysis and machine intelligence, vol. 36, no. 11, pp. 2227-2240, 2014.

[21] M. A. Fischler and R. C. Bolles, "Random sample consensus: a paradigm for model fitting with applications to image analysis and automated cartography," in Readings in computer vision. Elsevier, 1987, pp. 726740.

[22] H. Cantzler, "Random sample consensus(Ransac)," Institute for Perception, Action and Behaviour, Division of Informatics, pp. 2-5, 2005. [Online]. Available: http://citeseerx.ist.psu.edu/viewdoc/ download?doi=10.1.1.106.3035 $\{\&\}$ rep=rep $1\{\&\}$ type=pdf

[23] E. S. R. I. Inc. (2009) georeferencing a raster dataset. [Online]. Available: https://goo.gl/gu6Tur 